Article

\title{
Microanalysis, Pharmacokinetics and Tissue Distribution of Polysaccharide-Protein Complexes from Longan Pulp in Mice
}

\section{Ting Min ${ }^{1}$, Jie Sun ${ }^{1}$, Yang Yi ${ }^{1,2, *}$, Hong-Xun Wang ${ }^{1,2}$, Fei Hang ${ }^{3}$, You-Wei Ai ${ }^{1}$ and Li-Mei Wang ${ }^{1,2}$}

1 College of Food Science \& Engineering, Wuhan Polytechnic University, Wuhan 430023, China; E-Mails: minting1323@163.com (T.M.); qiqijiayuguan@163.com (J.S.); wanghongxunhust@163.com (H.-X.W.); aywlingyun@126.com (Y.-W.A.); wanglimeiyx@163.com (L.-M.W.)

2 Hubei Collaborative Innovation Center for Processing of Agricultural Products, Wuhan 430023, China

3 Sericultural \& Agri-food Research Institute, Guangdong Academy of Agricultural Sciences, Key Laboratory of Functional Foods, Ministry of Agriculture, Guangzhou 510610, China; E-Mail: hf1311@163.com

* Author to whom correspondence should be addressed; E-Mail: yiy86@whpu.edu.cn; Tel./Fax: +86-27-8391-3403.

Academic Editor: Chang Won Choi

Received: 4 August 2015 / Accepted: 8 October 2015 / Published: 15 October 2015

\begin{abstract}
A high performance size exclusion-fluorescence detection (HPSEC-FD) method combined with fluorescein isothiocyanate (FITC) prelabeling was established for the microanalysis of polysaccharide-protein complexes from longan pulp (LPP). FITC-labeled LPP (LPPF) was fractionated by gel filtration chromatography. The weight-average molecular weight and FITC substitution degree of LPPF were $39.01 \mathrm{kDa}$ and $0.20 \%$, respectively. The HPSEC-FD calibration curves linear over the range of $1-200 \mu \mathrm{g} / \mathrm{mL}$ in mouse plasma, spleen and lung samples with correlation coefficients greater than 0.995 . The inter-day and intra-day precisions of the method were not more than $6.9 \%$, and the relative recovery ranged from $93.7 \%$ to $106.4 \%$. The concentration-time curve of LPPF in plasma following intravenous (i.v.) administration at $40 \mathrm{mg} / \mathrm{kg}$ body weight well fitted to a two-compartment model. LPPF rapidly eliminated from plasma according to the short half-lives $\left(t_{1 / 2 \alpha}=2.23 \mathrm{~min}, t_{1 / 2 \beta}=39.11 \mathrm{~min}\right)$ and mean retention times $\left(M R T_{0-t}=1.15 \mathrm{~h}\right.$, $M R T_{0-\infty}=1.39 \mathrm{~h}$ ). After administration over 5 to $360 \mathrm{~min}$, the concentration of LPPF in
\end{abstract}


spleen homogenate decreased from 7.41 to $3.68 \mu \mathrm{g} / \mathrm{mL}$; the concentration in lung homogenate decreased from 9.08 to $3.40 \mu \mathrm{g} / \mathrm{mL}$. On the other hand, the increasing concentration of LPPF fraction with low molecular weight in heart homogenate was observed.

Keywords: longan pulp; polysaccharide-protein complex; HPSEC-FD; pharmacokinetics; distribution

\section{Introduction}

Longan fruit (Dimocarpus longan Lour.) has been traditionally used in Chinese medicinal formulation for a long time, serving as a common agent to promote blood metabolism, soothe nerves, relieve insomnia and prevent amnesia [1,2]. The extraction, purification, structure and bioactivity of the polysaccharides and polysaccharide-protein complexes (major bioactive ingredients) of longan pulp have been well studied [2-8]. Longan polysaccharide-protein complexes (LPP) can significantly enhance the immune functions of mice, such as antibody production against chicken red blood cells, ConA-induced splenocyte proliferation, macrophage phagocytosis, natural killer (NK) cell cytotoxicity against YAC-1 lymphoma cells and cytokine secretion in serum [4,8]. However, the potential mechanism related to their pharmacokinetics and tissue distributions in experimental animals is still unclear. In most cases, polysaccharides are studied as the carrier moiety of micromolecular drug (or natural bioactive ingredient), however their in vivo mechanism have rarely been investigated due to a lack of specific microassay methods $[9,10]$.

Most of the published studies on the pharmacokinetics and tissue distribution of polysaccharides used analytical methods including fluorescence labeling combined with chromatography $[9,11,12]$, isotope labeling [13,14], spectrophotometry [15,16], fluorospectrophotometry [17,18] and biological assay [19]. In view of the intrinsic characteristics of analyte and the sensitivity and specificity of the analytical method, the combination of high performance size exclusion chromatography (HPSEC) with fluorescent prelabeling was thought to be the most feasible method for the quantitative determination of LPP, especially in biological samples. Fluorescein isothiocyanate (FITC), which could combine with amino groups through the isothiocyano residue under weak alkali conditions [12], might be an effective labeling agent for the binding protein of LPP. Accordingly, a HPSEC-fluorescence detection (HPSEC-FD) method for the quantitative determination of LPP was established based on FITC prelabeling and followed with an investigation of the pharmacokinetics and tissue distribution in mice.

\section{Results and Discussion}

\subsection{Purification of Longan Polysaccharide Complexes (LPP) and Fluorescein Isothiocyanate-Labeled} $L P P(L P P F)$

Crude polysaccharide-protein complexes from longan pulp pretreated with or without FITC were fractionated on a Sephadex G-100 gel column to obtain the main fraction named LPPF or LPP

(Figure 1). The signals detected at $490 \mathrm{~nm}$ (phenol-sulfuric acid assay) and at $280 \mathrm{~nm}$ (spectrophotometer) belonged to polysaccharides and proteins, respectively. LPP were identified by 
the simultaneous absorbance peaks at 490 and $280 \mathrm{~nm}$ [20,21]. FITC showed the characteristic peaks at 280.0 and $489.5 \mathrm{~nm}$ (data not shown). As seen in Figure 1, the absorbances of LPP at 280 and $489.5 \mathrm{~nm}$ both increased after treatment with FITC indicating that LPPF were the FITC labeled derivatives of LPP [22]. The weight-average molecular weights $(M \mathrm{w})$ of LPP and LPPF were estimated to be 37.52 and $39.01 \mathrm{kDa}$, respectively. Their molecular weight distributions exhibited no obvious difference (Figure 2).
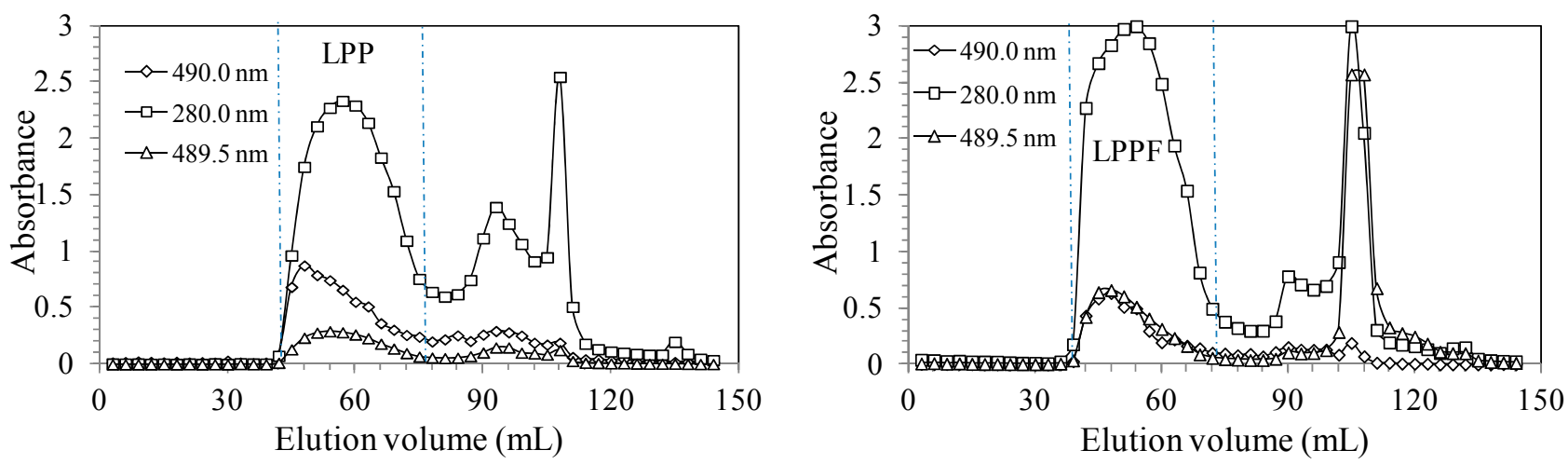

Figure 1. Gel filtration chromatograms of crude longan polysaccharide-protein complexes treated with (Right) or without (Left) Fluorescein isothiocyanate (FITC).
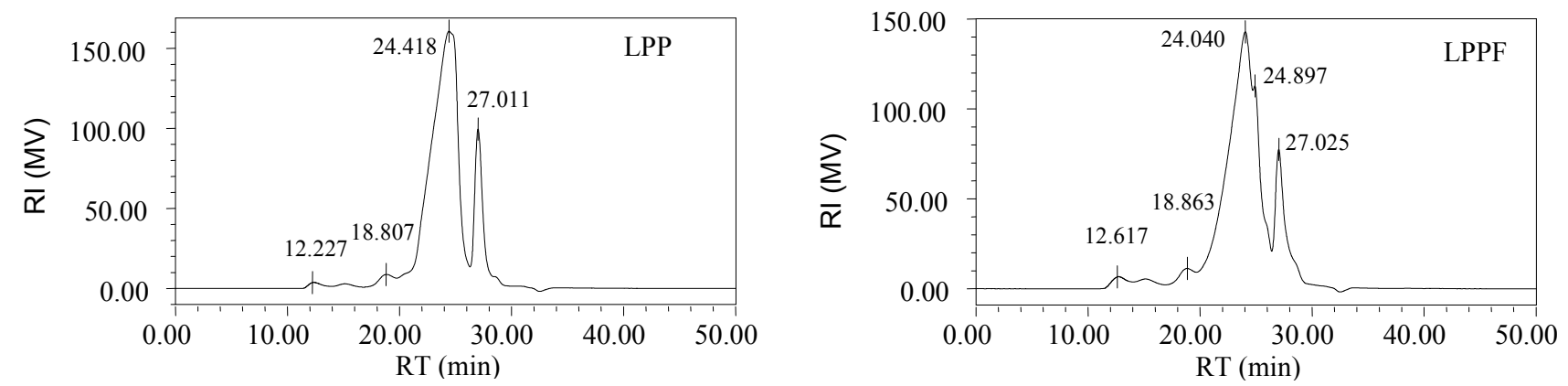

Figure 2. High performance size exclusion chromatograms of longan polysaccharide-protein complexes (LPP) (Left) and Fluorescein isothiocyanate-labeled LPP (LPPF) (Right) obtained by refractive index detection.

\subsection{Spectral Features of $L P P$ and $L P P F$}

As seen in the Ultraviolet-visible (UV-vis) spectra of LPP and LPPF (Figure 3), the absorbances of LPPF at about 490 and $280 \mathrm{~nm}$ were obviously stronger than those of LPP. It was consistent with the comparative analysis on their gel filtration chromatograms. In addition, only LPPF showed an evident fluorescence peak with peak value at $520 \mathrm{~nm}$. The emission wavelength and excitation wavelength for the HPSEC-FD method of LPPF were, respectively, 520 and $495 \mathrm{~nm}$, which were same as the FITC-labeled arabinogalactan and chitosan [9,23].

The linear regression equation of FITC concentration $(c, \mu \mathrm{g} / \mathrm{mL}) v s$. absorbance $(A)$ was established as follow: $A=0.202 c-0.003\left(R^{2}=0.999\right)$. The FITC substitution degree $(\%)$ of LPPF was calculated to be $0.20 \%$, which was significantly lower than that of FITC-labeled chitosan [23]. 

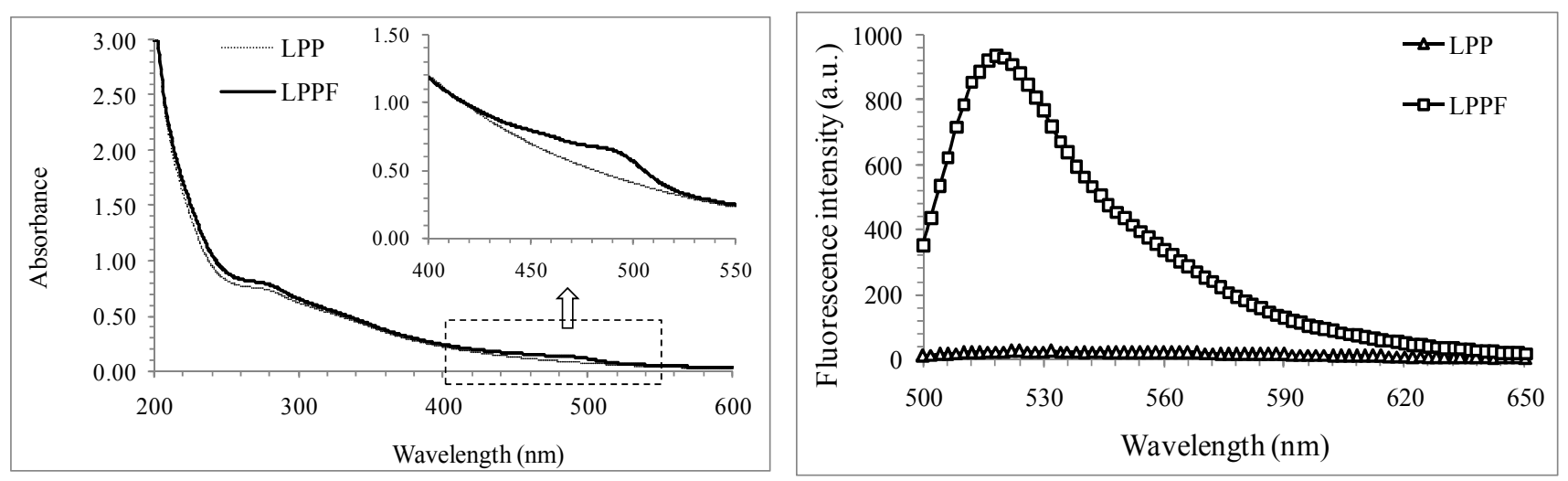

Figure 3. Ultraviolet-visible (Left) and fluorescence spectra (Right) of LPP and LPPF. The excitation wavelength for fluorescence scanning was $495 \mathrm{~nm}$.

\subsection{Method Validation}

Method specificity was demonstrated by assessing the interferences of endogenous substances from prepared biological samples. The chromatograms of LPPF obtained by FD in the presence and in the absence of interferences are shown in Figure 4. The typical peak value of LPPF was approximately $24.8 \mathrm{~min}$ (Figure 4A), which was consistent with the chromatogram obtained by refractive index detection (RID). The processed samples of mouse plasma, spleen and lung all exhibited no responsive signal in the chromatograms, i.e., they had no endogenous substance that significantly impacted on the detection of LPPF (Figure 4B,E,F). The characteristic peaks of other processed biological samples at about 28.1 min were partly overlapped with that of LPPF (Figure 4C,D,G). It was indicated that the proposed method could be only used for the microanalysis of LPPF in mouse plasma, spleen and lung. Likewise, the study of Lin et al. [11] also confirmed that blank plasma had no signal responding to fluorescence detection, but blank heart tissue sample had. FITC-labeled Ophiopogon japonicas polysaccharides [24] could be well-separated from the interferents of heart due to their lower molecular weights $(4.8 \mathrm{kDa})$ compared with LPPF. The biodisposition of FITC-labeled arabinogalactan in rats was investigated by detecting the fluorescence intensities of tissue samples [9], as well as that of FITC-labeled chitosan in mice [23]. However, the quantitative analyses of these polysaccharides might be interfered by the fluorescent substances derived from murine tissues including heart, liver and kidney. It was also suggested that the concentrations of FITC-labeled polysaccharides in mouse plasma, spleen and lung samples could be indirectly determined by the fluorescence intensity.

The standard curve of peak area ( $y$, arbitrary unit) to LPPF concentration $(x, \mu \mathrm{g} / \mathrm{mL})$ was constructed using the linear least squares regression model. The standard curves, correlation coefficients and linear ranges of quantitative determination of LPPF in the samples of plasma, spleen and lung are listed in Table 1. The HPSEC-FD method of LPPF showed good linear responses in the concentration range of $1-200 \mu \mathrm{g} / \mathrm{mL}$, as the correlation coefficients $\left(R^{2}\right)$ were larger than 0.995 . The limit of detection (DL) and the limit of quantization (QL) were 0.25 and $0.85 \mu \mathrm{g} / \mathrm{mL}$, respectively. The values were lower than those of phenol-sulfuric acid method (DL $1.54 \mu \mathrm{g} / \mathrm{mL}$, QL $4.67 \mu \mathrm{g} / \mathrm{mL}$ ) [25], but were higher than those of the HPSEC-FD method of FITC-labeled Radix Ophiopogonis polysaccharide due to the relatively lower FITC substitution degree of LPPF [11]. 

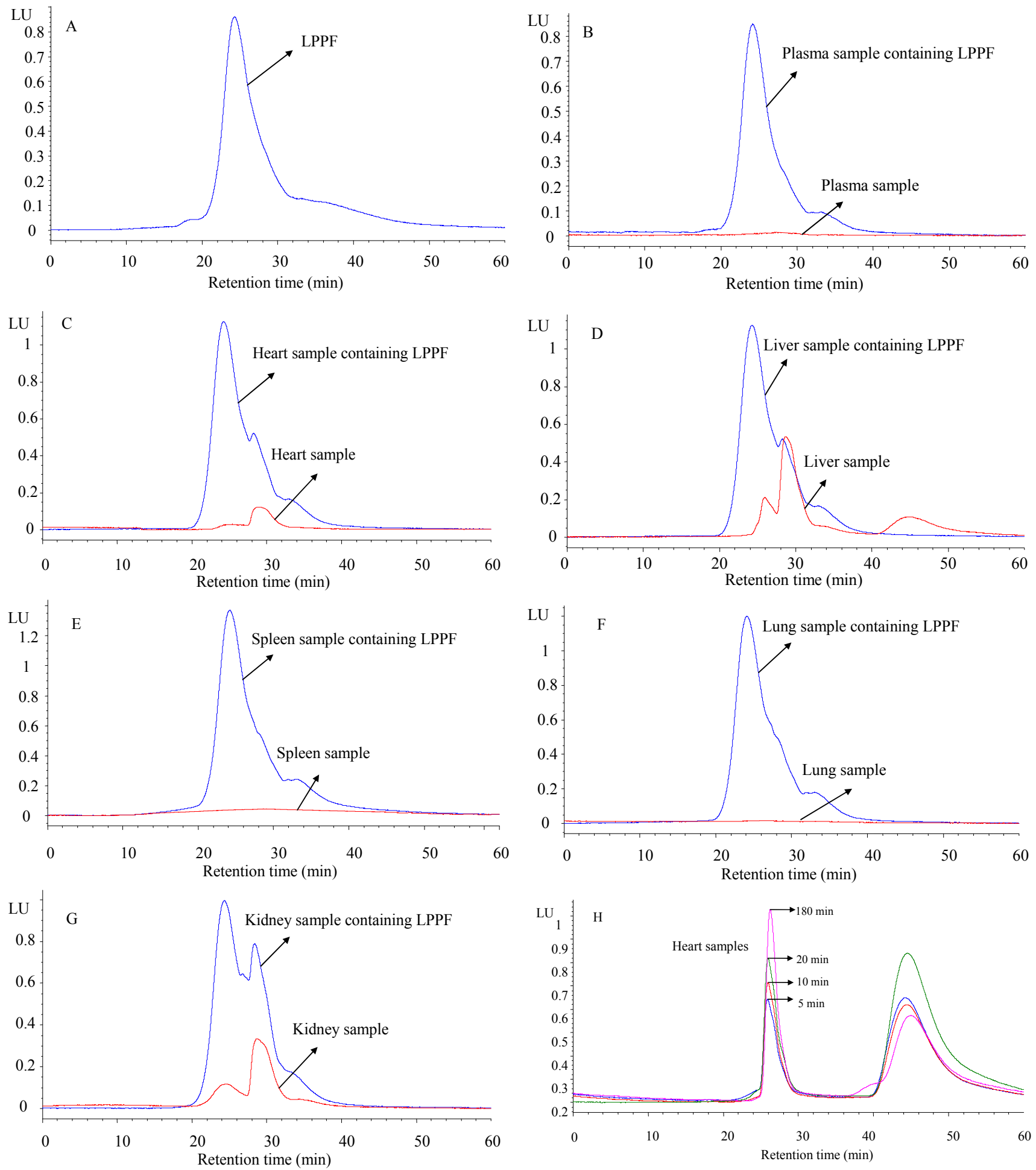

Figure 4. High performance size exclusion chromatograms of LPPF obtained by FD. (A) The chromatogram of $50 \mu \mathrm{g} / \mathrm{mL}$ LPPF prepared by phosphate buffer ( $\mathrm{pH} 7.4$ ); (B-G) The chromatograms of biological samples mixed with or without $50 \mu \mathrm{g} / \mathrm{mL}$ LPPF; (H) The chromatograms of heart samples from mice at predefined times $(5,10,20$ and $180 \mathrm{~min}$ ) after a single intravenous (i.v.) administration of LPPF (40 mg/kg body weight). 
Table 1. Calibration curves for Fluorescein isothiocyanate-labeled longan polysaccharide-protein complexes (LPPF) in mouse plasma and tissue homogenate supernatants.

\begin{tabular}{cccc}
\hline Biological Samples & Standard Curves & Correlation Coefficients & Linear Ranges * $(\boldsymbol{\mu g} / \mathbf{m L})$ \\
\hline Blank & $y=2.199 x+2.273$ & 0.998 & $1-200 \mu \mathrm{g} / \mathrm{mL}$ \\
Plasma & $y=2.223 x+1.034$ & 0.996 & $1-200 \mu \mathrm{g} / \mathrm{mL}$ \\
Spleen & $y=2.349 x-0.485$ & 0.996 & $1-200 \mu \mathrm{g} / \mathrm{mL}$ \\
Lung & $y=2.138 x+2.616$ & 0.995 & $1-200 \mu \mathrm{g} / \mathrm{mL}$ \\
\hline
\end{tabular}

* Data were obtained at six concentrations, and each repeated in triplicate.

Precision of the proposed method was explored by evaluating the peak areas of different concentrations of LPPF, to measure the agreement between test results from multiple and repeated instrumental analysis. As shown in Table 2, the intra-day precision and inter-day precision were basically in accordance with testing requirement $(R S D<5 \%)$. Accuracy evaluated by recovery test can measure the amount of analyte that is quantified relative to the amount present in the sample. The relative recovery values ranging from $93.7 \%$ to $106.4 \%$ were acceptable (Table 2) [11].

Table 2. Precision and accuracy for quantitative determination of LPPF by high performance size exclusion-fluorescence detection (HPSEC-FD) method.

\begin{tabular}{ccccc}
\hline Biological & Added Concentration & Relative & \multicolumn{2}{c}{ Precision $(\boldsymbol{R S D}, \boldsymbol{\%})$} \\
\cline { 4 - 5 } Samples & $(\boldsymbol{\mu g} / \mathbf{m L})$ & Recovery $(\boldsymbol{\%})$ & Intra-Day & Inter-Day \\
\hline \multirow{3}{*}{ Blank } & 2 & $99.4 \pm 4.3$ & 3.4 & 4.0 \\
& 40 & $101.7 \pm 3.0$ & 2.4 & 2.9 \\
& 80 & $101.4 \pm 2.3$ & 3.1 & 4.2 \\
\hline \multirow{3}{*}{ Plasma } & 2 & $98.2 \pm 5.1$ & 4.5 & 4.5 \\
& 40 & $102.3 \pm 4.1$ & 3.2 & 2.9 \\
& 80 & $103.4 \pm 5.2$ & 5.1 & 2.6 \\
\hline \multirow{3}{*}{ Spleen } & 2 & $106.4 \pm 3.9$ & 3.6 & 3.5 \\
& 40 & $96.7 \pm 5.7$ & 4.4 & 5.0 \\
& 80 & $105.4 \pm 3.3$ & 5.7 & 3.5 \\
\hline \multirow{3}{*}{ Lung } & 2 & $93.7 \pm 2.9$ & 3.0 & 4.2 \\
& 40 & $104.2 \pm 3.1$ & 4.4 & 3.8 \\
\hline
\end{tabular}

\subsection{Pharmacokinetics and Tissue Distribution of LPPF}

The concentration-time curve of LPPF in mice plasma following i.v. administration at $40 \mathrm{mg} / \mathrm{kg}$ body weight well fitted to a two-compartment model $\left(R^{2}=0.99 \pm 0.01, n=5\right)$. LPPF injected intravenously might be first distributed from central compartment into peripheral compartments to a pseudo-equilibrium (alpha phase), followed by a process of gradual elimination from plasma primarily attributed to excretion (beta phase), resulting in the biphasic decrease of plasma concentration of LPPF as shown in Figure 5. The important pharmacokinetics parameters of LPPF are summarized in Table 3. The concentration-time curves of bioactive polysaccharides in animal plasma were mostly confirmed to be two-compartment model, but their pharmacokinetics parameters showed significant differences due to different physicochemical characteristics, administration methods and dosages [10]. A typical two-compartment 
model of lentinan $(50 \mathrm{kDa})$ in mice plasma after intravenous administration was also identified [26]. In addition, the half-lives of distribution phase and elimination phase ( $t_{1 / 2 \alpha}$ and $\left.t_{1 / 2 \beta}\right)$ of lentinan in beagle dogs after i.v. administration showed positive correlations with dosage [27]. It was suggested that the reason for the longer $t_{1 / 2 \alpha}(1.27 \mathrm{~h})$ and $t_{1 / 2 \beta}(2.64 \mathrm{~h})$ of lentinan in mice plasma might be related to the much lower administration dosage $(0.5 \mathrm{mg} / \mathrm{kg}$ body weight), compared with LPPF. The results from clinical investigations indicated that most of the polysaccharides used for anti-cancer drug precursor had the $M \mathrm{w}$ ranging from 25 to $50 \mathrm{kDa}$, and the reasons for such a situation were closely related to the anti-cancer activity and pharmacokinetic behavior of polysaccharide [28]. Polysaccharides with larger $M_{\mathrm{w}}$ usually exhibited lower clearances and longer mean retention times (MRT) [10,29-31]. The correlation of molecular size to clearance and mean retention time may partially explain why lentinan had longer mean retention times $\left(M R T_{0-\mathrm{t}}=2.78 \mathrm{~h}, M R T_{0-\infty}=3.01 \mathrm{~h}\right)$ compared with LPPF [26], while Radix Ophiopogonis polysaccharide (4.8 kDa) showed a shorter one (39.0 min) [11].

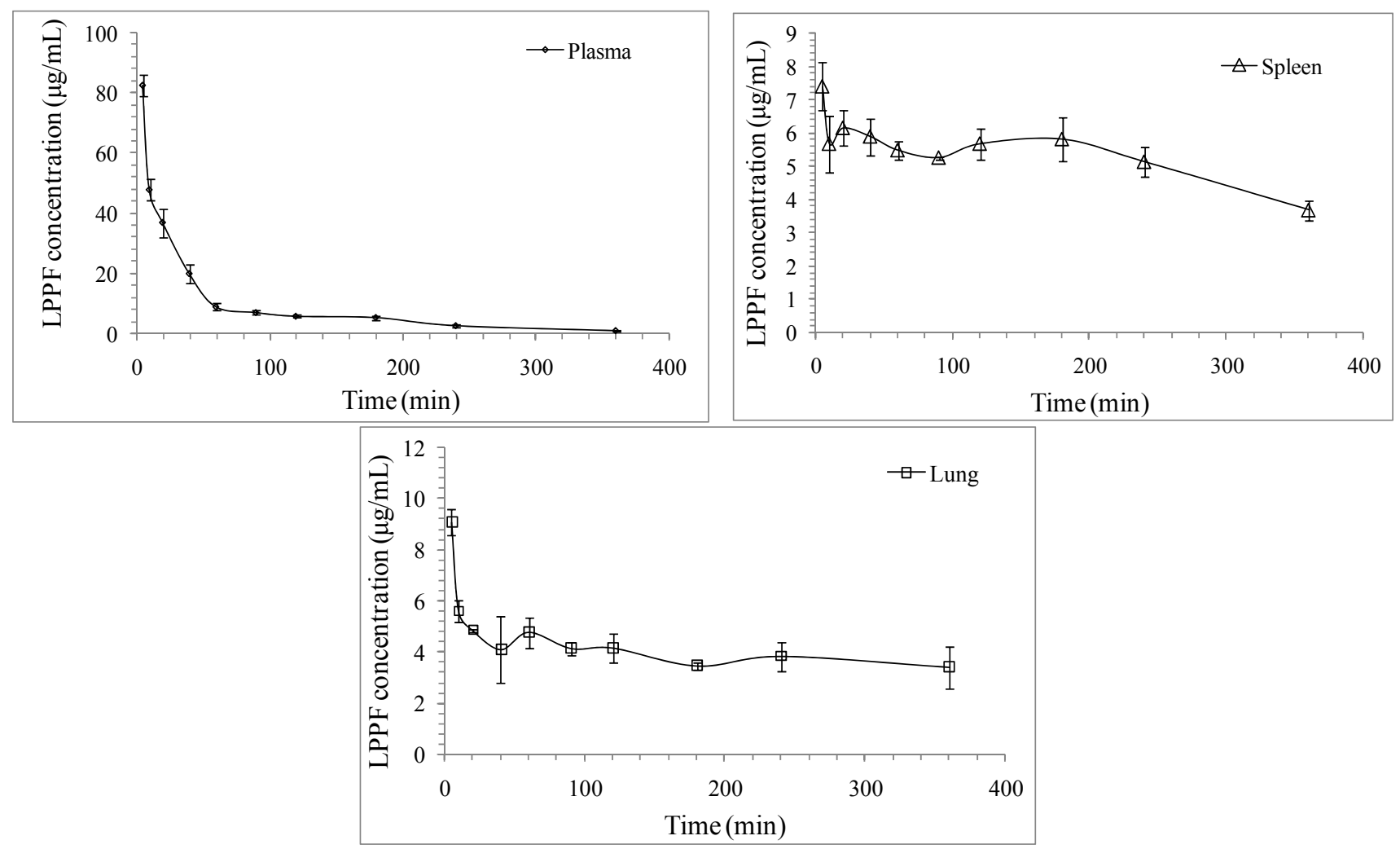

Figure 5. LPPF concentration $v s$. time profile following intravenous (i.v.) administration at $40 \mathrm{mg} / \mathrm{kg}$ body weight. Data are represented as mean \pm standard derivation $(n=5)$.

LPPF exhibited short half-lives in plasma, implying that LPPF could be rapidly transferred to peripheral tissues or excreted in urine. As shown in Figure 5, the LPPF concentration of spleen homogenate supernatant was measured to be $7.41 \mu \mathrm{g} / \mathrm{mL}$ at $5 \mathrm{~min}$ after administration, and then significantly decreased $(p<0.05)$. The concentrations ranging from 10 to 240 min exhibited no obvious difference $(p>0.05)$, but were all significantly higher than that at $360 \min (p<0.05)$. The LPPF concentration-time curve of lung homogenate supernatant was similar to that of spleen homogenate supernatant, as the highest value was $9.08 \mu \mathrm{g} / \mathrm{mL}$ at $5 \mathrm{~min}$ after administration. The results were close to those of Radix Ophiopogonis polysaccharide [11], and LPPF might be mainly distributed to kidney. 
The LPPF concentration in kidney could not be measured in the present study. However, deep yellow urine, which might contain a high concentration of LPPF, was repeatedly observed on mice sacrificed at 5 and $10 \mathrm{~min}$ after administration. The pharmacokinetics and biodisposition of polysaccharides were closely related to their molecular weights and net charges [10]. LPPF and LPP showed no significant difference in molecular weight. The charge only impacted on the uptake of polysaccharide by hepatocytes and tumor cells [10]. It could be concluded that the effect of FITC on the pharmacokinetics and biodisposition of LPP was negligible.

As seen in Figure 4H, the content of LPPF in heart might gradually increase after administration, according to the chromatograms of heart samples obtained at predefined times $(5,10,20,180 \mathrm{~min})$. This situation was different with the reports from Lin [11] and Zhang [26]. The factions of LPPF accumulated in heart showed smaller $M \mathrm{w}$, as the retention time of peak value (25.90 min) was in accordance with the $\mathrm{M} \mathrm{w}$ of $1.41 \mathrm{kDa}$. According to the appearance of new peak at about retention time $45 \mathrm{~min}$, the binding FITC of LPPF was speculated to be partly dissociated in heart. Chitosans with different $M_{\mathrm{W}}(112.3$ and $496.2 \mathrm{kDa}$ ) exhibited different tissue distributions in rats [18]. A few studies confirmed that the liver accumulation and kidney excretion of polysaccharides were related to $M_{\mathrm{w}}[16,32,33]$; polysaccharide selectivity of heart responding to $M \mathrm{w}$ was rarely reported.

Table 3. Pharmacokinetics parameters of LPPF after a single intravenous (i.v.) administration at $40 \mathrm{mg} / \mathrm{kg}$ (mean $\pm \mathrm{SD}, n=5$ ).

\begin{tabular}{cc}
\hline Parameters * & Values \\
\hline Half-life of distribution phase $t_{1 / 2 \alpha}(\mathrm{min})$ & $2.23 \pm 0.35$ \\
Half-life of elimination phase $t_{1 / 2 \beta}(\mathrm{min})$ & $39.11 \pm 2.73$ \\
Apparent volume of distribution of the central compartment $V_{1}(\mathrm{~L} / \mathrm{kg})$ & $0.09 \pm 0.01$ \\
Apparent volume of distribution of the peripheral compartment $V_{2}(\mathrm{~L} / \mathrm{kg})$ & $0.26 \pm 0.03$ \\
Clearance of the central compartment $C L_{1}(\mathrm{~L} / \mathrm{h} / \mathrm{kg})$ & $0.53 \pm 0.03$ \\
Clearance of the peripheral compartment $C L_{2}(\mathrm{~L} / \mathrm{h} / \mathrm{kg})$ & $0.60 \pm 0.05$ \\
Area under concentration-time curve $A U C_{0-\mathrm{t}}(\mathrm{mg} \cdot \mathrm{h} / \mathrm{L})$ & $56.80 \pm 3.44$ \\
Area under concentration-time curve $A U C_{0-\infty}(\mathrm{mg} \cdot \mathrm{h} / \mathrm{L})$ & $58.85 \pm 3.51$ \\
Mean retention time $M R T_{0-\mathrm{t}}(\mathrm{h})$ & $1.15 \pm 0.04$ \\
Mean retention time $M R T_{0-\infty}(\mathrm{h})$ & $1.39 \pm 0.08$ \\
\hline
\end{tabular}

* The parameters including $t_{1 / 2 \alpha}, t_{1 / 2 \beta}, V_{1}, V_{2}, C L_{1}$ and $C L_{2}$ were calculated by a two-compartmental moment analysis, and the other parameters were calculated by non-compartmental moment analysis.

\section{Experimental Section}

\subsection{Materials}

Fresh longan fruits (cv. Chu-Liang) were provided by Sericulture \& Agri-Food Research Institute of Guangdong Academy of Agricultural Sciences (Guangzhou, China). Longan pulps were manually stripped and hot-air dried at $75^{\circ} \mathrm{C}$ for $50 \mathrm{~h}$. The dried pulps were stored at $-20^{\circ} \mathrm{C}$ until use. 


\subsection{Animals and Biological Sample Preparation}

Eight weeks old Kunming mice (specific pathogen-free, male) were purchased from Lab Animal Center of Huazhong University of Science and Technology (Wuhan, China), and were kept in an environmentally controlled breeding room for a week before experiments. They were freely fed with standard laboratory food and water. All relative protocols and procedures were approved by our Institutional Animal Care and Use Committee, and were performed in accordance to the Guide for the Care and Use of Laboratory Animals.

Mice were sacrificed by bloodletting via eyeball to collect blood into a $1.5 \mathrm{~mL}$ heparin-soaked Eppendorf tube. After centrifugation at $3000 \mathrm{rpm} / \mathrm{min}$ for $10 \mathrm{~min}$, the separated plasma was frozen at $-20{ }^{\circ} \mathrm{C}$ until analysis. Various tissues including heart, liver, spleen, lung and kidney were immediately harvested. Each organ was homogenized with a glass homogenizer (6 cm diameter) using a 3-fold volume of $0.1 \mathrm{~mol} / \mathrm{L}$ phosphate buffer $(\mathrm{pH}$ 7.4) [11]. The homogenate was then centrifuged at $10,000 \mathrm{rpm} / \mathrm{min}$ for $5 \mathrm{~min}$ to collect supernatant. The supernatant was stored at $-20^{\circ} \mathrm{C}$ until analysis.

\subsection{Polysaccharide-Protein Complex Preparation}

Crude polysaccharide-protein complexes of longan pulp were isolated according to our previous method [6]. One hundred and fifty milligram complexes were dissolved in $10 \mathrm{~mL}$ sodium carbonate buffer solution $(0.5 \mathrm{~mol} / \mathrm{L}, \mathrm{pH} 8.3)$ with or without $10 \mathrm{mg}$ FITC [22]. After keeping the mixture in dark at room temperature for $24 \mathrm{~h}$, supernatants were separated by centrifugation at $4500 \mathrm{rpm} / \mathrm{min}$ for $10 \mathrm{~min}$. Three milliliter supernatants were injected onto a Sephadex G-100 gel column $(1.6 \mathrm{~cm} \times 50 \mathrm{~cm}$, gel was purchased from Pharmacia Biotech (Piscataway, NJ, USA)), followed by the elution with distilled water at a flow rate of $0.3 \mathrm{~mL} / \mathrm{min}$. Three milliliter per tube of eluants were collected for determining the concentrations of polysaccharide, protein and FITC by phenol-sulfuric acid method $(490 \mathrm{~nm})$ [34] and spectrometry (280 and $489.5 \mathrm{~nm})$, respectively. The eluants containing the main fraction, which was identified by gel filtration chromatogram, were vacuum-concentrated at $55{ }^{\circ} \mathrm{C}$ using a rotary evaporator (BC-R203, Shanghai Biochemical Equipment Co., Shanghai, China) and then freeze-dried to obtain FITC-labeled or unlabeled longan polysaccharide-protein complexes (i.e., LPPF and LPP). The yield of LPP from the crude complexes was $74.78 \%$. The polysaccharide content and protein content of LPP were $96.54 \%$ and $2.94 \%$, respectively.

\subsection{Spectrum Analysis}

Polysaccharide-protein complexes were dissolved in distilled water to the concentration of $0.5 \mathrm{mg} / \mathrm{mL}$. After centrifugation at $4500 \mathrm{rpm} / \mathrm{min}$ for $15 \mathrm{~min}$, the supernatant was scanned in the wavelength range of 200-600 nm using a UV-vis spectrophotometer (UV 1800, Shimadzu Corporation, Kyoto, Japan) and in the emission wavelength range of 500-700 $\mathrm{nm}$ (excitation wavelength $490 \mathrm{~nm}$ ) using a fluorescence spectrophotometer (FP-6500, Jasco Corporation, Kyoto, Japan).

A series of FITC solutions with concentrations in the range of $0.1-5.0 \mu \mathrm{g} / \mathrm{mL}$ was prepared by distilled water. Distilled water was used for zero setting. The absorbance of FITC solution was measured at $489.5 \mathrm{~nm}$ by a UV-vis spectrophotometer. The standard curve and regression equation of FITC concentration $v s$. absorbance were established. The absorbance difference between $50 \mu \mathrm{g} / \mathrm{mL}$ LPPF and 
$50 \mu \mathrm{g} / \mathrm{mL}$ LPP was used in the regression equation to calculate the concentration of binding FITC. The FITC substitution degree (\%) of LPPF was expressed as the mass percentage of binding FITC to LPPF.

\subsection{HPSEC Analysis}

\subsubsection{Detection Conditions}

HPSEC analysis was performed with a Waters Series system containing a refractive index detector (2414), a fluorescence detector (2475), a binary pump (1525) and an Ultrahydrogel 1000 SEC column (7.8 $\mathrm{mm} \times 300 \mathrm{~mm}$, Waters, Milford, MA, USA). The excitation wavelength and emission wavelength of FD were 495 and $520 \mathrm{~nm}$, respectively. Moreover, mobile phase was $0.1 \mathrm{~mol} / \mathrm{L}$ sodium nitrate, flow rate was $0.4 \mathrm{~mL} / \mathrm{min}$, injection volume was $25 \mu \mathrm{L}$, and column temperature was $35^{\circ} \mathrm{C}$.

Polysaccharide samples were dissolved in $0.1 \mathrm{~mol} / \mathrm{L}$ sodium nitrate to the concentration of $2 \mathrm{mg} / \mathrm{mL}$. The solutions were injected onto the SEC column after filtrating through a $0.45 \mu \mathrm{m}$ filter membrane and then detected using the refractive index detector. To estimate the $M \mathrm{w}$ of LPP and LPPF, poly(ethylene oxide) standards with known $M_{\mathrm{W}}\left(2.42 \times 10^{4}, 4.13 \times 10^{4}, 6.75 \times 10^{4}, 1.49 \times 10^{5}\right.$ and $3.13 \times 10^{5} \mathrm{kDa}$, Waters, Milford, MA, USA) were used for calibration.

\subsubsection{Selectivity}

LPPF samples were dissolved in phosphate buffer ( $\mathrm{pH} 7.4$ ) to the concentration of $500 \mu \mathrm{g} / \mathrm{mL}$. Twenty microliter LPPF solutions were added to $180 \mu \mathrm{L}$ blank plasma or $180 \mu \mathrm{L}$ tissue homogenate supernatant. The mixture was then treated according to the modified method of Kaneo et al. [9]. In brief, $200 \mu \mathrm{L}$ of the mixture were mixed with $80 \mu \mathrm{L}$ of $30 \%(w / v)$ trichloroacetic acid. After centrifugation at $14,000 \mathrm{rpm} / \mathrm{min}$ for $5 \mathrm{~min}$, the supernatant $(200 \mu \mathrm{L})$ was neutralized by addition of $30 \mu \mathrm{L} 11 \%(w / v)$ of $\mathrm{NaOH}$. After filtration, the biological sample containing or not containing $50 \mu \mathrm{g} / \mathrm{mL}$ LPPF was analyzed by the HPSEC-FD method.

\subsubsection{Quantitative Determination}

A series of standard LPPF solutions with concentrations in the range of $10-2000 \mu \mathrm{g} / \mathrm{mL}$ were prepared by phosphate buffer ( $\mathrm{pH}$ 7.4). Twenty microliter LPPF solutions were mixed with $180 \mu \mathrm{L}$ blank plasma or $180 \mu \mathrm{L}$ tissue homogenate supernatant and treated with the method as described in Section 3.5.2 to obtain the standard calibration samples with six levels ranging from 1 to $100 \mu \mathrm{g} / \mathrm{mL}$. The peak areas and retention times were used to establish a linear standard curve and the linearity correlation coefficient $\left(R^{2}\right)$ was calculated by regression analysis. DL and QL were calculated as the lowest level of LPPF that resulted in a signal-to-noise ratio of 3 and 10, respectively.

\subsubsection{Precision and Accuracy}

To evaluate the precision and accuracy of the method, repeatability (intra-day measurements) and intermediate precision (inter-day measurements) were examined by adding known levels of LPPF $(4,40$ and $80 \mu \mathrm{g} / \mathrm{mL})$. Repeatability was calculated from five replicate determinations of sample solution containing $20 \mu \mathrm{g} / \mathrm{mL}$ LPPF on same day. Intermediate precision was analyzed by the same experiment 
additionally repeated on three consecutive days. Precision and accuracy were calculated using the formulas described by Skidana et al. [35].

\subsection{Animal Experiment}

The mice, weighing 22-26 g, were fasted for $12 \mathrm{~h}$ prior to experiments. LPPF samples were dissolved in saline to the final concentration of $5 \mathrm{mg} / \mathrm{mL}$ for injection after filtration through a $0.45 \mu \mathrm{m}$ filter membrane. Mice were administrated with a single dose $(40 \mathrm{mg} / \mathrm{kg}$ body weight) of LPPF solution through caudal vein, and then were sacrificed by bloodletting via eyeball at predefined times $(5,10,20$, 40, 60, 90, 120, 180, 240 and $360 \mathrm{~min} ; n$ = five per time point) to collect bloods, spleens and lungs. Tissue homogenates were prepared by the procedures described by Lin et al. [11]. Twenty microliter phosphate buffer solutions were mixed with $180 \mu \mathrm{L}$ plasma or $180 \mu \mathrm{L}$ tissue homogenate supernatant. The mixture was then pretreated with the modified method of Kaneo et al. (as described in Section 3.5.2) [9] for HPSEC determination.

\subsection{Data Analysis}

Data were expressed as means \pm standard derivation (SD). Significance of difference $(p<0.05)$ was evaluated with one-way ANOVA followed by the Student-Newman-Keuls test using IBM SPSS Statistics 19 software (IBM, Armonk, NY, USA). The DAS 2.0 pharmacrokinetic program (Chinese Pharmacology Society, Shanghai, China) was used to calculate pharmacokinetic parameters.

\section{Conclusions}

The combination of FITC prelabeling and HPSEC-FD makes the quantitative determination of LPP possible in mouse plasma, spleen and lung samples. Based on the HPSEC-FD method, a typical two-compartment model of LPPF in mice plasma after intravenous administration was identified, and a small amount of LPPF were confirmed to be distributed to spleen and lung. Because of the impact of endogenous interferences from other tissues on the microanalysis method, the distribution of LPPF in mice has not been clearly explored. The proposed HPSEC-FD method needs to be modified for better sensitivity and specificity. Moreover, the effects of structure on the pharmacokinetics and tissue distribution of polysaccharide-protein complexes from longan pulp should be further investigated.

\section{Acknowledgments}

We gratefully acknowledge the financial support by National Natural Science Foundation of China (31301416).

\section{Author Contributions}

Conceived and designed the experiments: Yang Yi and Hong-Xun Wang. Performed the experiments and analyzed the data: Jie Sun, Yang Yi, You-Wei Ai, and Ting Min. Wrote the paper: Ting Min, Li-Mei Wang, and Fei Hang. 


\section{Conflicts of Interest}

The authors declare no conflict of interest.

\section{References}

1. Park, S.J.; Park, D.H.; Kim, D.H.; Lee, S.; Yoon, B.H.; Jung, W.Y.; Lee, K.T.; Cheong, J.H.; Ryu, J.H. The memory-enhancing effects of Euphoria longan fruit extract in mice. J. Ethnopharmacol. 2010, $128,160-165$.

2. Yang, C.; He, N.; Ling, X.; Ye, M.; Zhang, C.; Shao, W.; Yao, C.; Wang, Z.; Li, Q. The isolation and characterization of polysaccharides from longan pulp. Sep. Purif. Technol. 2008, 63, 226-230.

3. Zhong, K.; Wang, Q. Optimization of ultrasonic extraction of polysaccharides from dried longan pulp using response surface methodology. Carbohydr. Polym. 2010, 80, 19-25.

4. Zhong, K.; Wang, Q.; He, Y.; He, X. Evaluation of radicals scavenging, immunity-modulatory and antitumor activities of longan polysaccharides with ultrasonic extraction on in S180 tumor mice models. Int. J. Biol. Macromol. 2010, 47, 356-360.

5. Zhu, Q.; Jiang, Y.; Lin, S.; Wen, L.; Wu, D.; Zhao, M.; Chen, F.; Jia, Y.; Yang, B. Structural identification of $(1 \rightarrow 6)-\alpha$-D-glucan, a key responsible for the health benefits of longan, and evaluation of anticancer activity. Biomacromolecules 2013, 14, 1999-2003.

6. Yi, Y.; Zhang, M.W.; Liao, S.T.; Zhang, R.F.; Deng, Y.Y.; Wei, Z.C.; Tang, X.J.; Zhang, Y. Structural features and immunomodulatory activities of polysaccharides of longan pulp. Carbohydr. Polym. 2012, 87, 636-643.

7. Yi, Y.; Zhang, M.W.; Liao, S.T.; Zhang, R.F.; Deng, Y.Y.; Wei, Z.C.; Tang, X.J.; Zhang, Y. Effects of alkali dissociation on the molecular conformation and immunomodulatory activity of longan pulp polysaccharide (LPI). Carbohydr. Polym. 2012, 87, 1311-1317.

8. Yi, Y.; Liao, S.T.; Zhang, M.W.; Zhang, R.F.; Deng, Y.Y.; Yang, B.; Wei, Z.C. Immunomodulatory activity of polysaccharide-protein complex of longan (Dimocarpus longan lour.) pulp. Molecules 2011, 16, 10324-10336.

9. Kaneo, Y.; Ueno, T.; Tanaka, T.; Iwase, H.; Yamaguchi, Y.; Uemura, T. Pharmacokinetics and biodisposition of fluorescein-labeled arabinogalactan in rats. Int. J.Pharm. 2000, 201, 59-69.

10. Yi, Y.; Wang, H.-X.; He, J.-R. Research progresses of pharmacokinetics of polysaccharides. Acta Pharm. Sin. 2014, 49, 443-449.

11. Lin, X.; Wang, Z.; Sun, G.; Shen, L.; Xu, D.; Feng, Y. A sensitive and specific HPGPC-FD method for the study of pharmacokinetics and tissue distribution of Radix Ophiopogonis polysaccharide in rats. Biomed. Chromatogr. 2010, 24, 820-825.

12. Xie, Y. Label of Biomacromolecular Drugs and Their Pharmacokinetic Studies. Ph.D. Thesis, The Second Military Medical University, Shanghai, China, 2010.

13. Balogh, L.; Polyak, A.; Mathe, D.; Kiraly, R.; Thuroczy, J.; Terez, M.; Janoki, G.; Ting, Y.; Bucci, L.R.; Schauss, A.G. Absorption, uptake and tissue affinity of high-molecular-weight hyaluronan after oral administration in rats and dogs. J. Agric. Food Chem. 2008, 56, 10582-10593. 
14. Lendvai, N.; Casadevall, A.; Liang, Z.; Goldman, D.L.; Mukherjee, J.; Zuckier, L. Effect of immune mechanisms on the pharmacokinetics and organ distribution of cryptococcal polysaccharide. J. Infect. Dis. 1998, 177, 1647-1659.

15. De Lima, C.R.; Baccarin, R.Y.A.; Michelacci, Y.M. Reliability of 1,9-dimethylmethylene blue tests in comparison to agarose gel electrophoresis for quantification of urinary glycosaminoglycans. Clin. Chim. Acta 2007, 378, 206-215.

16. Guimarães, M.A.M.; Mourão, P.A.S. Urinary excretion of sulfated polysaccharides administered to wistar rats suggests a renal permselectivity to these polymers based on molecular size. Biochim. Biophys. Acta 1997, 1335, 161-172.

17. Ricea, P.J.; Lockharta, B.E.; Barkerb, L.A.; Adamsb, E.L.; Ensleyc, H.E.; Williams, D.L. Pharmacokinetics of fungal (1-3)- $\beta$-D-glucans following intravenous administration in rats. Int. Immunopharmacol. 2004, 4, 1209-1215.

18. Dong, W.; Han, B.; Shao, K.; Yang, Z.; Peng, Y.; Yang, Y.; Liu, W. Effects of molecular weights on the absorption, distribution and urinary excretion of intraperitoneally administrated carboxymethyl chitosan in rats. J. Mater. Sci. 2012, 12, 1-8.

19. Yoshida, M.; Roth, R.I.; Grunfeld, C.; Feingold, K.R.; Levin, J. Soluble (1 $\rightarrow 3)-\beta$-D-glucan purified from Candida albicans: Biologic effects and distribution in blood and organs in rabbits. J. Lab. Clin. Med. 1996, 128, 103-114.

20. Yi, Y.; Liao, S.T.; Zhang, M.W.; Shi, J.; Zhang, R.F.; Deng, Y.Y.; Wei, Z.C. Physicochemical characteristics and immunomodulatory activities of three polysaccharide-protein complexes of longan pulp. Molecules 2011, 16, 6148-6164.

21. He, L.; Ji, P.; Cheng, J.; Wang, Y.; Qian, H.; Li, W.; Gong, X.; Wang, Z. Structural characterization and immunostimulatory activity of a novel protein-bound polysaccharide produced by Hirsutella sinensis Liu, Guo, Yu \& zeng. Food Chem. 2013, 141, 946-953.

22. Lv, Z.H. Studies on Fluorescent Labeling and Pharmacokinetics of Marine Sufated Polysaccharide PS916. Ph.D. Thesis, Ocean University of China, Qindao, China, 2008.

23. Zeng, L.; Qin, C.; Wang, W.; Chi, W.; Li, W. Absorption and distribution of chitosan in mice after oral administration. Carbohydr. Polym. 2008, 71, 435-440.

24. Xu, D.-S.; Feng, Y.; Lin, X.; Deng, H.-L.; Fang, J.-N.; Dong, Q. Isolation, purification and structural analysis of a polysaccharide MDG-1 from Ophiopogon japonicus. Acta Pharm. Sin. 2005, 40, 636-639.

25. Wei, X.; Xi, X.; Wu, M.; Wang, Y. A novel method for quantitative determination of tea polysaccharide by resonance light scattering. Spectrochim. Acta Part A 2011, 79, 928-933.

26. Zhang, S. Studies on Pharmacokinetics and Tissue Distribution of Lentinan Liposome. Ph.D. Thesis, Huazhong University of Science and Technology, Wuhan, China, 2010.

27. Cai, Y. Studies on Pharmacodynamics and Pharmacokinetics of The lentinan Liposome. Ph.D. Thesis, Huazhong University of Science and Technology, Wuhan, China, 2009.

28. Haag, R.; Kratz, F. Polymer therapeutics: Concepts and applications. Angew. Chem. Int. Ed. 2006, 45, 1198-1215.

29. Peng, F. Pharmacokinetics Research on Bletillan Injections. Ph.D. Thesis, Soochow University, Suzhou, China, 2010. 
30. Lin, X.; Wang, Z.-J.; Huang, F.; Liang, S.; Shen, L.; Feng, Y.; Ruan, K.-F. Long-circulating delivery of bioactive polysaccharide from Radix Ophiopogonis by pegylation. Int. J. Nanomed. 2011, 6, $2865-2872$.

31. Lin, X.; Wang, Z.-J.; Wang, S.; Shen, L.; Feng, Y.; Ruan, K.-F.; Xu, D.-S. Comparison of tissue distribution of a pegylated Radix Ophiopogonis polysaccharide in mice with normal and ischemic myocardium. Eur. J. Pharm. Biopharm. 2011, 79, 621-626.

32. Kaneo, Y.; Uemura, T.; Tanaka, T.; Kanoh, S. Polysaccharides as drug carriers: Biodisposition of fluorescein-labeled dextrans in mice. Biol. Pharm. Bull. 1997, 20, 181-187.

33. Prescott, J.H.; Enriquez, P.; Jung, C.; Menz, E.; Groman, E.V. Larch arabinogalactan for hepatic drug delivery: Isolation and characterization of a $9 \mathrm{kDa}$ arabinogalactan fragment. Carbohydr. Res. 1995, 278, 113-128.

34. Dubois, M.; Gilles, K.A.; Hamilton, J.K.; Pebers, P.A.; Smith, F. Colorimetric method for determination of sugars and related substances. Anal. Chem. 1956, 28, 350-356.

35. Skidana, I.; Grunwaldb, J.; Thekkedatha, R.; Degterevc, A.; Torchilina, V. A HPLC method for the quantitative determination of $\mathrm{N}$-(2-hydroxy-5-nitrophenylcarbamothioyl)-3,5-dimethylbenzamide inbiological samples. J. Chromatogr. B 2011, 879, 1610-1616.

(C) 2015 by the authors; licensee MDPI, Basel, Switzerland. This article is an open access article distributed under the terms and conditions of the Creative Commons Attribution license (http://creativecommons.org/licenses/by/4.0/). 